\title{
BMJ Open Impact of a videoconferencing educational programme for the management of concurrent disorders on nurses' competency development and clinical practice: protocol for a convergent mixed methods study
}

\author{
Gabrielle Chicoine (D) , , ${ }^{1,2}$ José Côté, ${ }^{1,2,3}$ Jacinthe Pepin, ${ }^{1}$ Pierre Pluye, ${ }^{4}$ \\ Louise Boyer, ${ }^{1}$ Guillaume Fontaine (D) , ${ }^{1,5}$ Geneviève Rouleau (D) , ${ }^{3,6}$ \\ Simon Dubreucq, ${ }^{2,7}$ Didier Jutras-Aswad ${ }^{2,7}$
}

To cite: Chicoine G, Côté J, Pepin J, et al. Impact of a videoconferencing educational programme for the management of concurrent disorders on nurses' competency development and clinical practice: protocol for a convergent mixed methods study. BMJ Open 2021;11:e042875. doi:10.1136/ bmjopen-2020-042875

- Prepublication history and additional material for this paper are available online. To view these files, please visit the journal online (http://dx.doi. org/10.1136/bmjopen-2020042875).

Received 20 July 2020 Revised 12 January 2021 Accepted 02 February 2021

\section{Check for updates}

(C) Author(s) (or their employer(s)) 2021. Re-use permitted under CC BY-NC. No commercial re-use. See rights and permissions. Published by BMJ.

For numbered affiliations see end of article.

\section{Correspondence to} Mrs Gabrielle Chicoine; gabrielle.chicoine@umontreal. ca

\section{ABSTRACT}

Introduction Extension for Community Healthcare Outcomes (Project ECHOC) is an innovative model for continuing professional development that uses videoconferencing technology to support and train general practitioners remotely. The model has been replicated to a variety of settings and locations for capacity building in healthcare professionals caring for patients with chronic and complex health conditions. Limited research has been conducted so far on the impact of ECHO in the field of concurrent mental health and substance use disorders (ie, concurrent disorders (CDs)). Therefore, this mixed methods study aims to develop a comprehensive understanding of an ECHO programme impact for CD management on nurses' competency development and clinical practice. Methods and analysis The proposed mixed methods study, based on a convergent parallel design, will be conducted in the province of Quebec, Canada, to collect, analyse and interpret quantitative (QUAN) and qualitative (QUAL) data from a specific ECHO Program on CDs. In the QUAN component, an observational prospective cohort study will be conducted over a 12-month period. All nurses who participated in the programme between 2018 and 2020 and who consent to research will be recruited to collect data on the extent of their learning and practice outcomes at three time points. Alongside the surveys, nurses will be invited to participate in individual semistructured interviews. In-depth QUAL data will be subjected to a thematic analysis and will assist in exploring how and in which conditions nurses developed and mobilised their competencies in clinical practice. A comparison-of-results strategy will be used in the final integration component of the study.

Ethics and dissemination This study protocol was approved by the Ethics Committee of the Universite de Montréal Hospital Center (\#19.295) and the Université de Montréal Ethics Committee (CERSES-20-017 R). We aim to disseminate the findings through international academic conferences, international peer-reviewed journals and professional media.
Strengths and limitations of this study

- To the best of our knowledge, this is the first mixed methods study aiming to understand the long-term impact of an ECHO programme for CD management on nurses' competency development and clinical practice.

- In accordance with guidelines for reporting a mixed methods study, the proposed convergent parallel design will rely on a closely coordinated combination of methods and on an explicit wording of when and how integration will occur.

- Using an interpretive description approach, the qualitative method will expand on the process of competency development in the context of a videoconferencing educational programme, as well as facilitators and barriers to their mobilisation in clinical practice.

- The non-experimental nature of the quantitative method and the anticipated small sample size will limit the results' interpretation by preventing any causal association and generalisability.

- Guided by an innovative social constructionism conceptual framework, this mixed methods study will combine a diversity of outcomes and perceptions to make recommendations about how to effectively support nurses in acquiring a high level of competency.

\section{INTRODUCTION}

\section{Concurrent disorders (CDs)}

CDs refers to co-occurring mental health $(\mathrm{MH})$ and substance use disorders (SUD). ${ }^{1}$ In addition to their high prevalence, ${ }^{2} \mathrm{CDs}$ are frequently associated with higher rates of relapse, worse psychiatric symptoms, increased odds of unplanned rehospitalisation, poorer treatment compliance and 
prognosis compared with MH only. ${ }^{34}$ Furthermore, individuals with CDs often experience higher risks of suicide, violence or delinquency, cardiovascular illnesses, as well as transmitted infections. ${ }^{5}$ CDs are also correlated with poor psychosocial outcomes including homelessness, social isolation, stigma and incarceration. ${ }^{6}$ A typical example illustrating this clinical problem is a person suffering from a dual diagnosis of schizophrenia and opioid use disorder who also contracted the hepatitis C virus and uses cannabis on a regular basis. ${ }^{7}$ This complex relationship between MH, SUD and related psychosocial problems highlights the specific needs of individuals living with CDs, which can pose a significant challenge for healthcare professionals attempting to care for them. ${ }^{8}$

Despite evidence supporting the efficiency of integrated treatment of both MH and SUD, ${ }^{10}$ it has been suggested that individuals living with CDs have poor access to care. ${ }^{11}$ A report from the Substance Abuse and Mental Health Services Administration ${ }^{12}$ revealed that among American individuals living with CDs who sought health services in the last 12 months, only $7.4 \%$ of them received care for at least one of their conditions. Furthermore, it appears that $55 \%$ of the patients' group received no treatment at all. This gap in access to care is associated with issues in healthcare provision, for example, the lack of competent human resources and suggests a clear need to improve care and patients' outcomes. $^{13}$

\section{Nurses' competencies in caring for individuals with CDs}

As the largest professional group within $\mathrm{MH}$ and addiction primary care services, ${ }^{14}$ nurses are uniquely positioned to provide care for patients with CDs. Indeed, nurses are qualified in assessment and intervention delivery and spend most of their time in direct contact with patients. ${ }^{15}$ However, nurses' difficulties in caring for patients with MH problems who also problematically use alcohol or drugs have been highlighted in numerous studies. ${ }^{16-18}$ For example, Wener and Woodgate ${ }^{19}$ reported that feelings of ambivalence, frustration and powerlessness are common among Canadian primary care nurses who encounter individuals living with CDs. This situation is further exacerbated in rural and remote areas, ${ }^{20}$ whereas many nurses frequently feel ill-equipped and emotionally exhausted in addressing the care needs of complex and severe cases of patients with CDs. ${ }^{21} 22$

Consequently, caring for this challenging population can create a significant pressure on nurses, which has been associated with a higher vulnerability to burnout syndrome, low job satisfaction and poor employee retention. ${ }^{23-25}$ Research also indicates that nurses have suboptimal competencies for screening mental disorders in active users, offering appropriate interventions in crisis situations and coordinating care. ${ }^{1126}$ In addition, multiple studies have shown that nurses may hold negative or judgemental attitudes towards patients with CDs. ${ }^{17} 27$ Results from a systematic review $(n=28)$ indicated that healthcare professionals holding stigmatised attitudes towards SUD have a more task-oriented approach and perceived their knowledge and skills as inadequate. ${ }^{28}$

\section{Continuing professional education in CDs}

In light of the complexities related to CDs, combined with the challenges faced by professionals in providing appropriate care for this priority patient population, there has been a growing recognition that further educational opportunities are needed. ${ }^{29-31}$ A recent scoping review ( $n=32$ ) underlines the growing body of evidence with regard to the effectiveness of continuing education in improving attitudes, increasing knowledge and confidence and supporting clinical practice change in nurses working with individuals with $\mathrm{CDs} .{ }^{15}$ In this context, continuing education can be understood as a planned and systematic effort to support healthcare professionals in developing a higher level of competency in order to improve clinical performance and patients' health outcomes. $^{32}$

A potential intervention for developing nurses' competencies in CDs is Extension for Community Healthcare Outcomes (ECHO), a promising model in continuing education using videoconferencing (VC) technology to establish networks between specialists from centralised academic centres and other healthcare professionals. ${ }^{33}$ ECHO provides telementoring and opportunities for sharing best practices on the management of a wide range of topics and conditions. Ultimately, the programme seeks to reduce variation in care delivery and improve patients' health outcomes. The ECHO model considers factors affecting professionals' participation in continuing education activities such as cost, lack of time, long-distance travelling, ability to access and use technology and credential incentives. ${ }^{34}$ Thus, it constitutes an interesting, flexible and convenient online learning intervention. Indeed, the ECHO model offers professionals the opportunity to discuss real patient cases, share common realities with peers, practise what they have learnt in a safe environment and be positively reinforced.

A systematic review $(n=52)$ reported a positive impact of the ECHO model on healthcare professionals' outcomes such as satisfaction, knowledge and confidence.$^{35}$ Furthermore, there is growing evidence in support of the acceptability and feasibility of the ECHO programme, notably for reducing professionals' sense of isolation in rural settings and in terms of cost-effectiveness. ${ }^{36}$ There is also a body of research showing the ECHO model effectiveness in terms of providing comparable care to specialists in the case of hepatitis $\mathrm{C}$ treatment, ${ }^{37} 38$ chronic pain management $^{39-41}$ and geriatric care. ${ }^{42}{ }^{43}$ Despite those promising results, ${ }^{35} 36$ the evidence regarding the impact of ECHO in the field of CDs remains limited. For instance, we found only four studies reporting on $\mathrm{MH}$ and/or addiction-specific ECHO programmes. ${ }^{44-47}$ In three of these studies, increases in professionals' knowledge using objective measures and self-efficacy using survey rating scales were reported after a 6 -month, ${ }^{46}{ }^{47}$ and 8-month 
periods. ${ }^{45}$ However, these results were statistically significant only in Mehrotra et al. ${ }^{47}$

In conclusion, the few studies discussed previously included quantitative observational designs, of which we noted several limitations: small sample sizes, lack of longitudinal data, interpretation of findings independently of professional groups and lack of control for cofounders. ${ }^{3548}$ The majority of these studies contain insufficient information on how the implemented programme was replicated or adapted and how well it was delivered. ${ }^{48}{ }^{49}$ Although few studies have been conducted to assess the impact of ECHO on nurses, ${ }^{50}{ }^{51}$ these studies were not specific to CDs and have not adequately captured nurses' views on how and in which conditions they developed their competencies. Indeed, limited research has been conducted so far to explore ECHO's impact on competency development, and how these new acquisitions were applied into clinical nursing practice. Thus, there is a need to obtain quantitative (QUAN) results on the long-term impact and to enrich our comprehension of these results using detailed qualitative (QUAL) findings. The present study aims to bridge these gaps by developing a comprehensive understanding of an ECHO programme impact for CD management on nurses' competency development and clinical practice.

\section{Philosophical and conceptual foundations}

Ian Hacking's social constructionism, ${ }^{52}$ which emphasises a pluralistic-embedded ontological and epistemological inquiry, will guide this study. This worldview is well suited for planning, conducting and appraising mixed methods research. ${ }^{53}{ }^{54}$ Hacking's proposal for a social theory of science has inspired the development of a conceptual framework for the purpose of achieving a comprehensive understanding of a $\mathrm{VC}$ educational programme impact for CD management on nurses' competency development and clinical practice. Our conceptualisation stipulates that studying the impact of a programme implies the combination of different forms of evidence, each of them producing a mixed kind of evidence using a looping effect (feedback effect). This conceptualisation is illustrated in figure 1 and summarised below.

In accordance with Gregor's typology, ${ }^{55}$ our conceptual framework provides a description (how we will understand) of the phenomena of interest (the programme impact), the categories of outcomes of continuing education (Moore et $a l \mathrm{~s}^{56}$ first five levels of assessment) and the main concepts (competency development and their mobilisation in clinical practice).$^{57}$ Outcomes and concepts will be described using QUAN and QUAL evidence, respectively. The framework represents the related inputs (data source, collection and analysis), the interactions between the constructs' 'outcomes and competencies' (looping effect between QUAN and QUAL evidence) and how these might be understood (integration of QUAN and QUAL methods). This conception is innovative because it builds on existing and traditional evidence of the ECHO model, while it also suggests an alternative perspective to the current dominance of 'gapspotting' in the literature. ${ }^{58}$

\section{METHODS AND ANALYSIS}

The proposed study seeks to answer the following three overarching research questions:

- Q1. QUAN question: what is the evolution in nurses' outcomes over a 12-month period of participation in a Canadian ECHO programme for CD management?

- Q2. QUAL question: how and in which conditions nurses mobilised in their clinical practice the competencies they perceived to have developed through their participation?

- Q3. Mixed QUAN and QUAL question: in what ways the evolution in nurses' outcomes over a 12-month

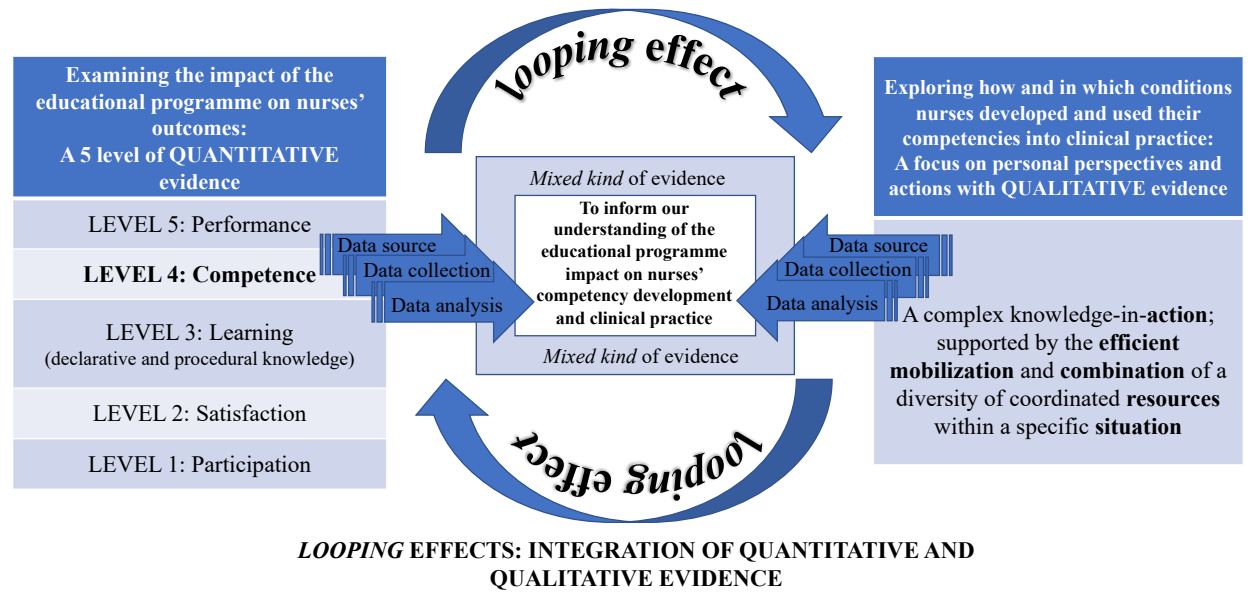

Figure 1 Integrative conceptual framework for studying the impact of a videoconference educational programme for the management of concurrent disorders on nurses' competency development and clinical practice. ${ }^{525657}$ 


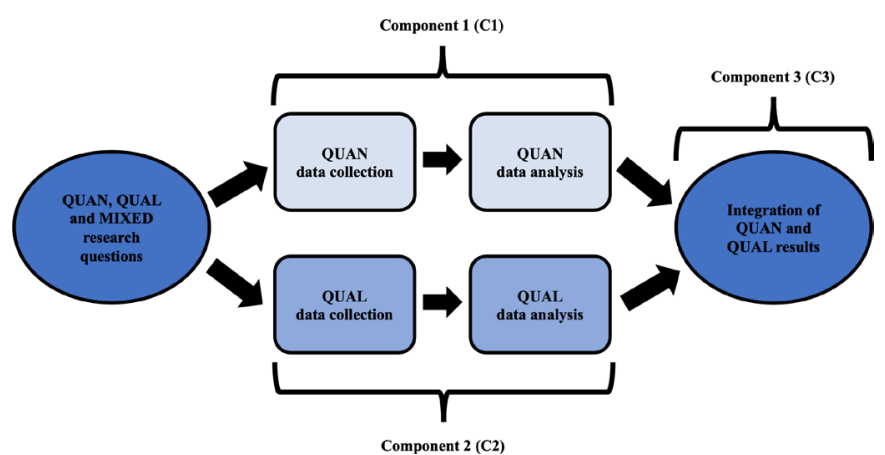

Figure 2 Visual model for the mixed methods convergent parallel design. QUAL, qualitative; QUAN, quantitative.

period of participation in a Canadian ECHO programme for $\mathrm{CD}$ management is related to the development and mobilisation of their competencies in clinical practice?

This protocol was organised according to the Good Reporting of A Mixed Method Study checklist, ${ }^{59}$ the Strengthening the Reporting of Observational studies in Epidemiology guidelines, ${ }^{60}$ the Consolidated Criteria for REporting Qualitative research checklist ${ }^{61}$ and Phillips et $a l \mathrm{~s}^{62}$ guideline for Reporting Evidence-based practice Educational interventions and Teaching. The items of the checklists are fulfilled in online supplemental file 1.

\section{Study design}

This study will use a mixed methods convergent parallel design for the purpose of complementarity and comparison between QUAN and QUAL methods. ${ }^{63}$ We will collect and analyse QUAN and QUAL data simultaneously and independently. Then, we will combine QUAN and QUAL results in the final integration of the study (see figure 2). Integration-the merging or dialogue between the QUAN and QUAL component of the study-will allow the QUAL results to give meaning and depth to the QUAN results collected over a long period. ${ }^{64}$ In the integration component of the study, the knowledge yield via QUAN and QUAL methods undertaken independently will achieve a 'whole greater than the sum of the parts'. ${ }^{64}$ In other words, we do not aim to explain QUAN results and do not need to wait for them to undertake the QUAL component of the study.

As shown in figure 2, the planned convergent parallel design involves three components, C1, C2 and C3, which address the research questions Q1, Q2 and Q3, respectively.

(C1) QUAN data collection and analysis: Among nurses who participated in a Canadian ECHO programme for CD management, we intend to examine the evolution of nurses' outcomes over a 12-month period. This will be achieved using an observational prospective single group cohort study design (QUAN method).

(C2) QUAL data collection and analysis: we will conduct individual semistructured interviews using an interpretive description approach ${ }^{65}$ with nurses who participated in the ECHO programme. We aim to explore the competencies that nurses perceive to have developed in CDs and to enlighten our understanding of how and in which conditions they mobilised these competencies in their clinical practice (QUAL method).

(C3) Integration of QUAN and QUAL results: the integration aims to merge the QUAN and QUAL methods. ${ }^{54}$ The final product of the integration, which is also the final result of the study, will be obtained by considering the insights gained from merging nurses' outcomes on the programme impact (QUAN results) with nurses' perceptions of their competency development (QUAL results).

\section{Setting, VC educational programme and technical features}

Based on the ECHO model, this study will use VC technology to connect professionals across multiple settings and disciplines in real-time educative sessions with an interdisciplinary team of experts in CDs. The programme was developed in 2017 and implemented in September 2018 in a Canadian tertiary hospital in the province of Quebec. One of the promising elements of the programme is the group modality, according to the premise that social interactions are essential conditions for effective learning to occur. ${ }^{66}$ Thus, the ECHO model endorses that sharing professional experiences improves and reinforces learning, while peer support enhances one's motivation and self-perception of being able to perform new behaviours. ${ }^{67}$ To consolidate learning, opportunities for practice and feedback in authentic settings are also provided.

The educative programme is organised in scheduled virtual sessions with the Zoom platform (Zoom Video Communications Inc, 2016), which allows professionals to connect with and see each other in real time from their work or home environment. PowerPoint presentations can be shared throughout the sessions, and a chat forum is also available to participants. To run a virtual session online, each participant needs a desktop or a laptop computer (Windows, macOS or Linux), a reliable internet connection (broadband wired or wireless), speakers and a microphone (built-in, USB plug-in or wireless Bluetooth) and a webcam or HD cam (built-in, USB pug-in, an HD cam or HD camcorder with a videocapture card). Participants also have the possibility to join the virtual sessions from their tablet, phone or any mobile device. Prior to the beginning of the programme, information and tips about running a Zoom session are provided to participants by email. Before each virtual session, an encrypted Zoom meeting link with an encryption used is sent only to registered participants to prevent hacking of the platform. A computer scientist is also available in person for the expert team and online for other participants to resolve any technical issues. Registered participants are not required to hold a Zoom licence to run the virtual sessions online. More information about VC equipment and other technical features of the VC setting can be found in Phillips et al's ${ }^{62}$ guideline (see online supplemental file 1). 
Each curriculum of the programme includes a total of 20 sessions delivered over a 10-month period (SeptemberJune). The sessions last 1 hour and a half and are held at 2-week intervals. During each session, a professional or a team of professionals present a patient case for management guidance. The case-based discussions are supplemented with expert recommendations and short didactic presentations on a disease-related topic that are both tailored to professionals' needs along the curriculum (see online supplemental file 2).

\section{C1: quantitative method}

\section{Participants and recruitment}

All nurses who participated in the ECHO programme on CDs for the 2018-2019 and 2019-2020 curricula will be invited to participate in the study $(n=65)$. Nurses will be eligible for inclusion if they hold a valid licence of practice. Participation will be considered if nurses have attended at least one virtual session during an entire curriculum. This will ensure that eligible participants have a minimal exposition of the educative intervention, ${ }^{35}$ without excluding those who participated more passively and may potentially benefit from the program. ${ }^{68}$

\section{Outcomes and measurements}

We intend to collect a number of outcomes as part of a self-administered online survey (SurveyMonkey, 20192020) including nurses' participation, satisfaction and acceptability regarding the programme, attitudes towards patients with CDs, knowledge in CDs, self-efficacy in CDs management and perceived clinical performance. ${ }^{56}$ Sociodemographic and practice information will also be collected. Table 1 summarises the QUAN outcomes' description and operationalisation, with corresponding sources of data to be captured.

A visual representation of when each outcome will be measured during the study is shown in online supplemental file 3. At each data collection point, a link to the survey will be provided to nurses, and they will be asked to complete it within a 3-week period. The instruments to be used in the survey were developed with a panel of experts in CDs and inspired from previous studies on the ECHO programme. ${ }^{33} 45$ Further detail regarding each instrument is provided in online supplemental file 4. QUAN data from nurses will be collected at three time points (before attending the programme (T0), 6 (T1) and 12 (T2) months after the first programme attendance). The developed survey was pilot tested with a small sample of healthcare professionals prior to its use in the study. ${ }^{69}$

\section{Sample size consideration}

We aimed for a sample size of 30 participants with completed questionnaires at T0, T1 and T2. Considering the total available population $(\mathrm{n}=65)$ and previous ECHO studies recruitment rates, we assume: (1) $60 \%$ of the total hospital nurse population will be recruited and will complete the baseline questionnaire; (2) $90 \%$ of those recruited will remain in the study at 6 months and will complete the evaluation questionnaires; and (3) $90 \%$ of those who participated at 6 months will remain in the study and will complete the final 12-month questionnaires. As this study is descriptive and exploratory, we do not plan to test any specific statistical hypothesis and will not make sample size estimates from a power analytic perspective. The sample size of 30 will allow us to estimate the binary outcomes of participation with no more than $18 \%$ precision using the normal approximation to the $95 \%$ CI.

\section{Statistical analysis plan}

We will use simple univariate descriptive statistics to evaluate the ECHO programme impact. The available sociodemographic and the evolution in nurses' outcomes (participation, satisfaction, knowledge, attitudes, selfefficacy and clinical performance) at each time point will be calculated. For continuous variables, descriptive statistics (mean, SD, median, IQR, minimum and maximum) will be provided. For categorical variables, the number of patients and the percentages will be reported. Paired t-test will be used for changes from baseline measures. If we have enough data, the bivariate analyses will examine the sociodemographic and professional level factors associated with study outcomes. The evaluation in time for all longitudinal variables will be presented in graphical form. The effect sizes of the ECHO programme impact with $95 \%$ CI will be extracted from the respective statistical models. The SAS V.9.4 statistical software will be used for all calculations (SAS Institute Inc, 2020).

\section{C2: qualitative method}

\section{Participants and recruitment}

All nurses with a valid licence of practice who participated in the ECHO programme between 2018 and 2020 for at least one virtual session and who consent to research will be included in the QUAL component. Each potential participant will be recruited by the first author (GC) through email or by phone, according to their preferred contact information. According to Thorne ${ }^{65}$ data saturation is not a desired outcome in the interpretive description approach since practical disciplines such as nursing tend to appreciate that subjective experience can theoretically possess infinite variation. Instead, interpretive description focuses on obtaining a deeper understanding of participants' perspective while recognising that variation in perceptions may exist. ${ }^{70}$ Considering that QUAL strategies for data collection are time consuming and based on prior studies in the field of nursing education, ${ }^{70}$ we anticipate that $50 \%$ of the whole population $(\mathrm{n}=65)$ will agree in taking part of the QUAL component $(n= \pm 32)$. However, sample size will be evaluated on an ongoing basis as participants volunteer: by noting nurses' characteristics (ie, registration year in the ECHO programme, academic background, past and current clinical experiences and healthcare settings) and by ensuring that the data gathered from participants are rich enough to answer the QUAL research question (Q2). 
Table 1 QUAN outcomes' description and operationalisation, with corresponding sources of data

\section{Assessment levels and desirable outcomes in Moore et al's ${ }^{56}$ framework \\ Outcomes measurement in this study}

Level $1 \quad$ Participation

Participation

Satisfaction articipation

\section{Corresponding sources of QUAN data}

- Attendance records within the last 6 months.

- Learning objectives and motivations for attending the programme.

- Number of virtual sessions in which nurses interacted (verbal interaction or within the chat forum) with the other participants within the last 6 months.

- Number of virtual sessions in which nurses presented a patient case within the last 6 months.

- Perceived level of participation within the last 6 months.

Satisfaction and acceptability regarding the $\mathrm{ECHO}$ programme

$\rightarrow A$ A self-reported questionnaire including measurement of the degree of nurses' satisfaction and acceptability regarding the programme. The questionnaire comprises the following dimensions: quality of the information (2), quality of the technological infrastructure (1), general satisfaction (2) and perceived usefulness of the programme (8). ${ }^{81}$

\begin{tabular}{|c|c|c|c|c|}
\hline $\begin{array}{l}\text { Level } 3 \mathrm{~A} \\
\text { Declarative knowledge }\end{array}$ & $\begin{array}{l}\text { Declarative and } \\
\text { procedural knowledge: } \\
\text { Attitudes towards } \\
\text { patients with CDs. }\end{array}$ & $\begin{array}{l}\text { A French version of the Comorbidity } \\
\text { Problems Perceptions Questionnaire } \\
\text { to measure nurses' attitudes towards } \\
\text { patients with CDs. }\end{array}$ & 33 items & $\alpha=0.90^{83-85}$ \\
\hline $\begin{array}{l}\text { Level } 4 \\
\text { Competence }\end{array}$ & $\begin{array}{l}\text { Self-efficacy in CD } \\
\text { management. }\end{array}$ & $\begin{array}{l}\text { A self-reported questionnaire measuring } \\
\text { nurses' level of confidence in CDs } \\
\text { management. }{ }^{86} 87\end{array}$ & 19 items & $\begin{array}{l}\text { Study-specific } \\
\text { questionnaire }\end{array}$ \\
\hline $\begin{array}{l}\text { Level } 5 \\
\text { Performance }\end{array}$ & $\begin{array}{l}\text { Perceived clinical } \\
\text { performance }\end{array}$ & $\begin{array}{l}\text { A self-reported questionnaire } \\
\text { measuring: (1) the number of patients } \\
\text { with CDs seen or followed in the last } 6 \\
\text { months, (2) the number of patients with } \\
\text { CDs that nurses were able to manage } \\
\text { without referring them to a specialised } \\
\text { service in CDs and (3) the extent of } \\
\text { nurses' application of the experts' } \\
\text { recommendations and/or new learning } \\
\text { acquisitions in their clinical practice } \\
\text { within the last } 6 \text { months. }\end{array}$ & Three questions & $\begin{array}{l}\text { Study-specific } \\
\text { questionnaire }\end{array}$ \\
\hline $\begin{array}{l}\text { Level } 6 \\
\text { Patient health }\end{array}$ & NA & & & \\
\hline $\begin{array}{l}\text { Level } 7 \\
\text { Community health }\end{array}$ & NA & & & \\
\hline
\end{tabular}

Note: adapted from 'Achieving desired results and improved outcomes: integrating planning and assessment throughout learning activities', by D E Moore, J S Green and H A, Gallis, 2009, Journal of Continuing Education in the Health Professions, 29(1), p. 3. This study will not report on patient and community health outcomes. While Moore et al's ${ }^{56}$ framework has seven levels of assessment, this study will focus on the first five levels as part of an initial programme investigation. We have added attitudes towards people with CDs since they are known to play a central role in the quality of care delivery. ${ }^{22}$

$\alpha$, Cronbach's alpha; CDs, concurrent disorders; NA, not applicable; QUAN, quantitative.

\section{Semistructured interviews}

We will conduct individual semistructured interviews with the Zoom platform for the purpose of exploring nurses' perceptions of how they developed their competencies over their participation in the ECHO programme and how they mobilised them into clinical practice when 
attempting to care for individuals with CDs. We also intend to collect data on nurses' perceptions of the conditions that might have foster or hinder their competency development (eg, personal or contextual factors, educational programme components and technical setting). Interviews will allow us to investigate nurses' perceptions of the programme benefits for their competency development and to have the opportunity to explore how the programme could be further improved. Interviews will be guided by prompts as well as open-ended questions (see online supplemental file 5). Nurses will be encouraged to give significant examples from their clinical practice and to reflect on their clinical decisions and actions. Interviews will last approximately 1 hour and will be conducted by the first author (GC) at the time each participant will deem convenient.

\section{Qualitative data analysis}

QUAL data will be subjected to a thematic analysis. ${ }^{71}$ In interpretive description, data collection and analysis occur concurrently each informing the other in an iterative process. ${ }^{70}$ Therefore, the analytic process will begin after conducting a first interview and will approximately last 9 months. This will enable prolonged and close contact with the data. Once the interviews are transcribed by a researcher assistant, the first author (GC) will be listening to the audio recordings to familiarise herself with the data and to verify transcripts' accuracy. Data will be imported, coded and managed using the QUAL data analysis software tool MAXQDA Standard (MAXQDA Distribution by VERBI GmbH, 1995-2020).

The following step will be taken to scrutinise each interview transcript for meaningful units (quotations) and to generate initial codes. The codes will be subsequently classified under broad categories for further analysis. In each category, codes will be examined, and common themes among the codes will be searched to create a first set of themes representing the data patterns. The next step implicates to make sense of the data by reviewing the meaningful themes within each interview transcripts and across datasets. In this way, a hierarchy of themes pinpointing relationships between themes will first be created, and the themes will be refined and renamed until the emergent results contribute to answer Q2.

Confirmation of coding and themes will be performed at each stage of the process with two other researchers (JC and JP) in order to prevent researcher bias. Trustworthiness of the QUAL findings will also be validated using a member checking technique ${ }^{72}$ in which the analysed data (ie, emergent themes) will be returned to each participant to check for accuracy and resonance with his or her perceptions.

\section{C3: integration of QUAN and QUAL results}

In the integration component of the study, we will adopt a comparison strategy to combine and contrast the QUAN and QUAL results. ${ }^{54}$ In this strategy, the resource composed of the QUAN and QUAL results obtained

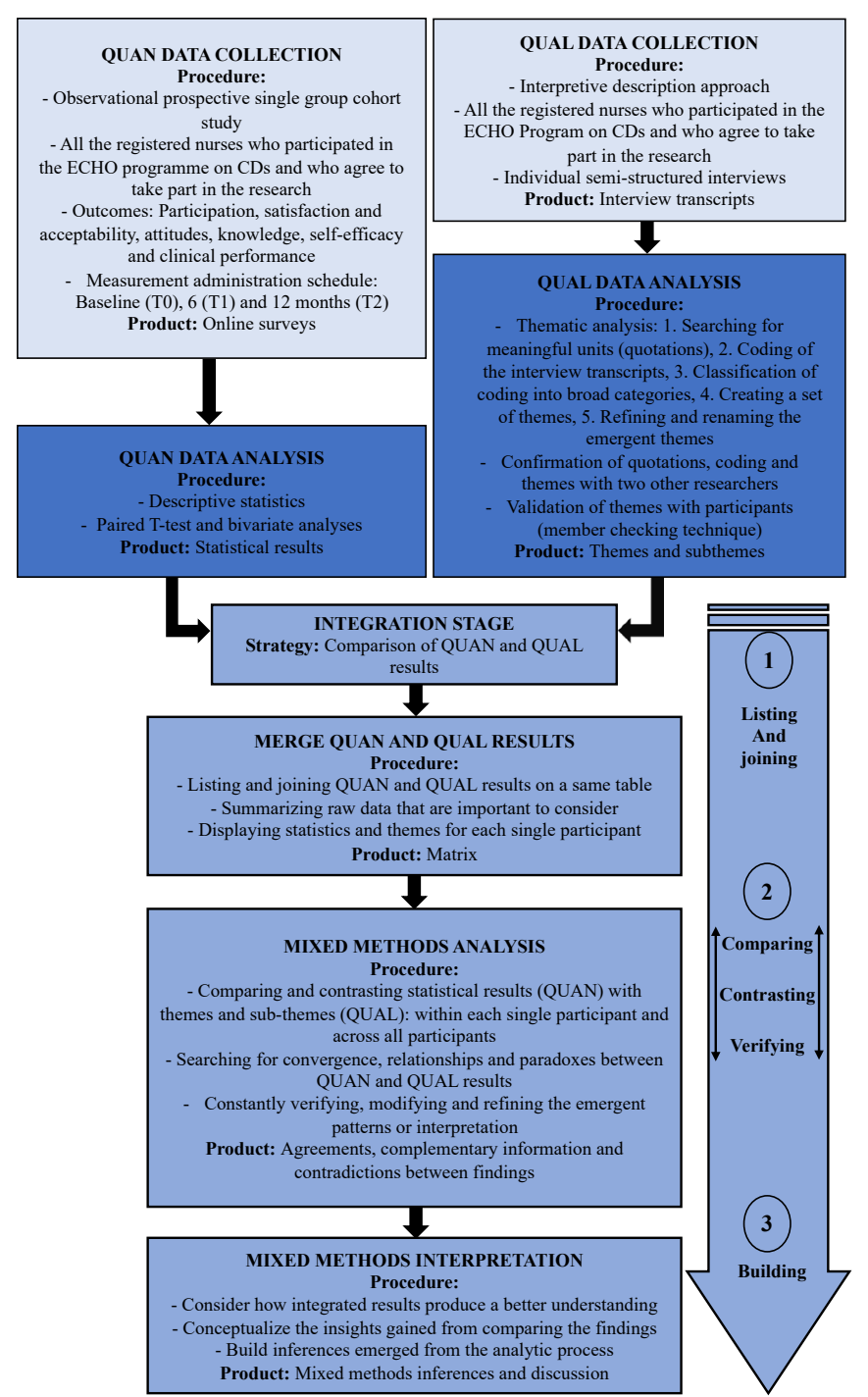

Figure 3 Mixed methods procedures for integrating QUAN and QUAL results using a comparison of results' strategy. ${ }^{54} 73$ CDs, concurrent disorder; ECHO, Extension for Community Healthcare Outcomes; QUAL, qualitative; QUAN, quantitative.

through separated data collection and analysis, and the final product constitute the mixed methods inferences. Figure 3 summarises the overall process and procedures to be applied in the comparison strategy. ${ }^{73}$

To achieve this comparison, we will use a matrix technique to summarise and display the QUAN and QUAL results, thereby allowing consideration of both methods simultaneously. ${ }^{74}$ This technique will assist in conducting a 'third effort' of analysis ${ }^{75}$ and deepen our understanding of the overall results. Statistical results from surveys and emergent themes from the interviews will be charted in order to concisely map the main study findings on the same page. Information will be organised in a results-byparticipant fashion. ${ }^{64}$ This organisation will allow us to pay attention in unexpected relationships and paradoxes between QUAN and QUAL results shared within a single 


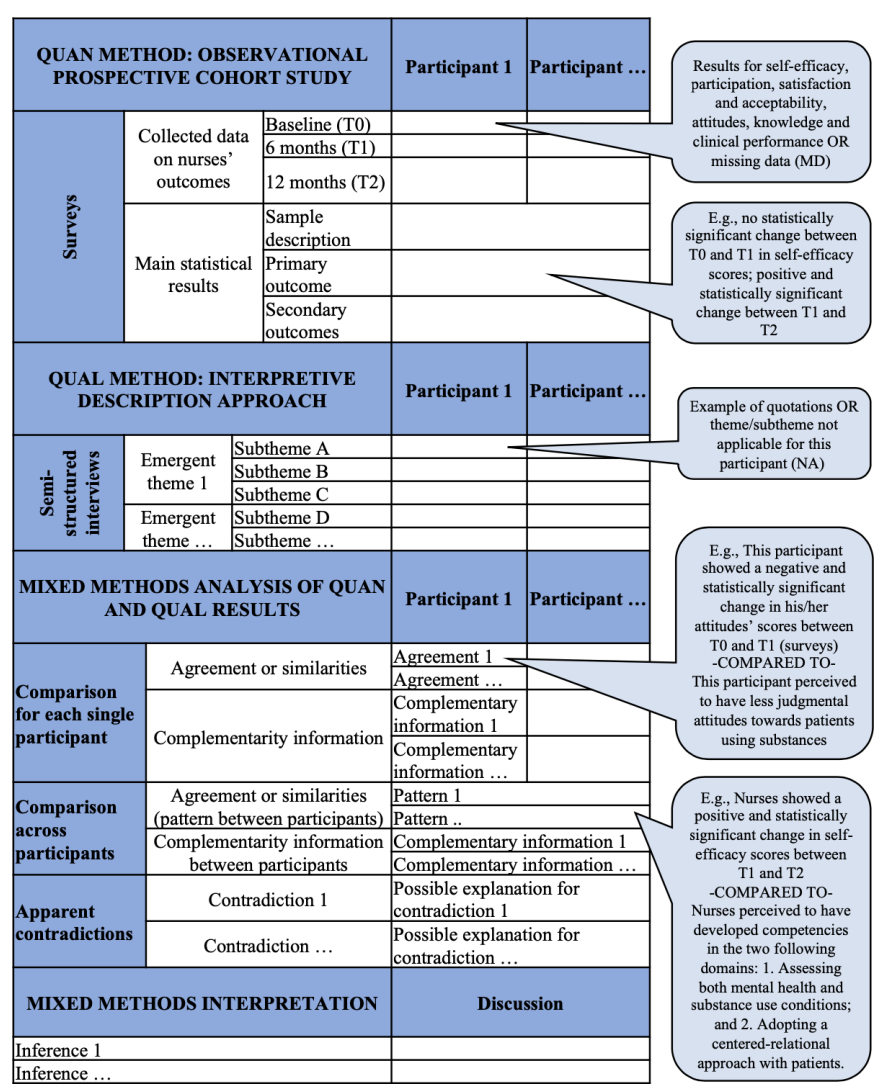

Figure 4 Example of a matrix to compare QUAN and QUAL results. QUAL, qualitative; QUAN, quantitative.

participant, and then search for patterns across all participants. ${ }^{76}$ Findings from each component of the study will be assessed for agreement (convergence and similarities) and complementarity. We will meticulously scrutinise any apparent divergences (contradiction, discrepancy or dissonance), and consider possible explanations to resolve them. ${ }^{77}$ Figure 4 shows an example of a matrix.

\section{Patient and public involvement}

Patients and the public will not be involved in the design of, recruitment for and conduct of this study. However, nurses will be invited to reflect on their appreciation of the programme content, structure and delivery (eg, didactic presentations, teaching methods, experts' recommendations, time allowed for case-based discussions, schedule, VC equipment, material and other resources) through an online survey after each virtual session (as part of the programme requirements to obtain continuing education credentials) and as part of the online satisfaction and acceptability questionnaire developed for this study. The programme has also implemented an 'ECHO participant committee' in which voluntary nurses are asked for suggestions and/or modifications in order to better adapt the programme to their learning and practice needs (ie, programme improvements). Education for healthcare professionals has been identified as a priority research area for its impact on the quality and security of care and relation to patients' outcomes. ${ }^{78}$ We expect that the study findings will inform on the conditions and strategies that are likely to strengthen the effectiveness of educational programmes in CDs.

\section{Current status}

This mixed methods study is an addition to an ongoing prospective observational cohort study for which ethical approval has been obtained in December 2018 from the Ethics Committee of the Université de Montréal Hospital Center (\#18.245). As of December 2020, we are currently collecting QUAN and QUAL data, and we will continue collecting through 2021.

\section{ETHICS AND DISSEMINATION Ethical considerations}

This study protocol was approved by the Ethics Committee of the Université de Montréal Hospital Center on 14 January 2020 (\#19.295) and from the Université de Montréal Ethics Committee (CERSES-20-017 R) on 30 January 2020. The proposed study follows the Canadian Tri-Council Policy Statement ethical guidelines ${ }^{79}$ as described in the paragraphs below.

First, written informed consent will be obtained from nurses for both QUAN and QUAL data collection. On registration to the ECHO programme, a first electronic consent form will be sent to nurses informing them on the nature and specific objectives of the QUAN component of the study. Nurses will also be asked their contact information and if they agree to be contacted for further research projects. This information will be stored in an encrypted and separated document on a secure server of the tertiary hospital research centre. The contact information will be maintained until the QUAL data collection stage, so that surveys can be sent and linked with responses and interviews' transcripts. Contact information will be removed and replaced with a random identification code after interview completion. A second electronic informed consent form will be sent to nurses engaging in the semistructured interviews. Before each interview, the first author (GC) will make sure that nurses are aware that the discussion will be audio recorded.

Second, several precautions will be taken to ensure that nurses understand that their participation in the study is voluntary and outside of the ongoing programme. Both consent forms will explain that nurses' participation is confidential and that they have the right to withdraw at any time throughout the study. We will also ensure that nurses are aware that refusing to participate will have no effect on their registration in the programme. Solicitation for the interviews will not take place during the videoconference sessions. This will ensure fairness between nurses and other healthcare professionals and distinction between the study and the programme. All the interviews will be conducted outside working hours, and nurses who accept to participate will be offered a $\$$ C50 voucher for their contribution.

Third, this study will collect a combination of electronic and material-based data that will be securely 
and safely stored throughout the study. All electronic data, including signed consent forms, survey responses, audio files and transcribed interviews, will be stored on a secured server. Answers from completed surveys will be uploaded from SurveyMonkey and organised in a protected-encrypted Excel document by a research assistant. Physical documentation including observation notes taken during the interviews will be exclusively kept within the hospital research centre in a locked drawer of a locked room. Only the first author (GC), members of the research team (JC, JP and DJ-A) and those involved in interview transcription will have access to the raw data. All electronic and material documents will be kept for a 7-year period and destroyed afterwards, in line with the ethics committee's policies. We also recognise that nurses may be anxious or fearful of reprisal when completing a survey or participating in an interview that explores their knowledge and competencies in CDs. However, nurses will be made aware that the collected data will in no way be used to assess employee performance.

Finally, a key issue concerning linking outcomes from the surveys with verbatim from transcripts interview is whether a participant is identifiable from the information collected. To manage this risk, sociodemographic and practices' outcomes will be collected and reported in broad categories (eg, area of practice will be collected instead of specific names of work settings). Any information that could identify a particular participant or setting during the interviews will be removed from transcripts. Also, the observation notes will not contain any information related to the participants' identity, as they will only be referred to by their role (eg, registered, assistant or specialist nurse). The thematic analysis will provide a certain level of abstraction within the raw data so that any association between emergent themes and participants will be prevented. Any identifiable information (ie, interview transcripts and quotations) will be retrieved from the final study report and will not be disseminated as per the plan described below.

\section{Dissemination plan}

The dissemination plan will include both traditional (ie, academic publication, scientific conferences and professional congresses) and innovative (eg, Project ECHO Institute software repository, links with local and regional agencies and web portals) means of ensuring the study findings are communicated locally, regionally and internationally. The plan will ensure that the results from this study are accessible to a broad audience and transdisciplinary communities including scholars, students, practitioners, citizens, policymakers and other stakeholders. A summary report will be shared through emails to the participating nurses and available online (http://www. ruis.umontreal.ca/CECTC_echo; https://labo-jutrasaswad.ca/). Finally, it is expected that the results of this study will be suitable for publication in relevant peerreviewed journals; we plan to prepare a first manuscript detailing the QUAL method and results and a second manuscript including the QUAN and MIXED components of the study.

\section{STRENGTHS AND LIMITATIONS}

This study will add to the existing body of evidence regarding the ECHO programme's impact on nurses' competency development and clinical practice. Also, we proposed an integrative conceptual framework to guide the proposed study, and we expect that this creative conceptualisation might shed new light on the ECHO programme's impact. A key strength of this study is that the planned mixed methods convergent design relies on a thoughtful, strategic and synergic use of two methods, which will enhance the overall comprehensiveness of the study results.

However, this study has limitations. Given the observational nature of the QUAN method, a single cohort study design was chosen. Hence, the absence of a control group will limit the QUAN results' interpretation at the description level, preventing any causal association between nurses' participation in the ECHO programme and the evolution in their learning and practice outcomes. In terms of generalisability, this study will focus on nurses so that findings may not be broadly applicable to other professional groups in contact with patients with CDs in their clinical practice. Since many of the instruments we intend to use in the surveys are not validated, it is possible that the measurements may not truly reflect the outcomes targeted by this study. Given that participation in this study is voluntary, a selection bias may exist as nurses who do not wish to participate may not have similar outcomes and/or perceptions that we will not be able to capture.

Other limitations include small sample size, loss to follow-up and missing data. Efforts to minimise loss to follow-up will include offering a $\$ \mathrm{C} 50$ voucher as acknowledgement of participants' contribution, multiple reminders and flexible hours for data collection. It is also possible that participants may over-rate the programme impact in terms of learning and practise benefits due to the presence of the researcher that would challenge the internal validity of the reported results. Nonetheless, these biases should be limited based on the breadth and depth of comprehensiveness that we intend to gain in mixing both QUAN and QUAL methods (corroboration of results).

Finally, we anticipate that divergences between QUAN and QUAL results are a potential mixed methods' threat to internal validity in the case of a convergent design. ${ }^{63}$ To address and reflect on this issue, the matrix technique will help to identify those contradictions and clarify how we will resolve them in a transparent and rigorous manner. From another angle, discrepancy can also generate unexpected discoveries, pave the way to new ideas and raise challenging questions that may contribute to further research and practice recommendations. Discussing how divergence issues will be approached throughout this mixed methods study will offer appropriate and 
meaningful guidance in conducting and reporting convergent designs and perhaps contribute to methodology advancement. ${ }^{80}$

Despite those limitations, we expect that this study will expand our current knowledge on how effectively the ECHO model supports nurses in developing higher levels of competency in CDs. Furthermore, this study will contribute to ensuring that new ECHO programmes in the field of CDs and future larger controlled studies assessing their efficacy are well designed. There is the potential both to provide guidance to educators, clinical leaders and researchers and ultimately to positively impact access to adequate care by people living with CDs.

\section{Author affiliations}

${ }^{1}$ Faculty of Nursing, Université de Montréal, Montreal, Quebec, Canada

${ }^{2}$ Research Center, Université de Montréal Hospital Center, Montreal, Quebec,

Canada

${ }^{3}$ Research Chair in Innovative Nursing Practices, Faculty of Nursing, Université de Montréal, Montreal, Quebec, Canada

${ }^{4}$ Department of Family Medicine, Faculty of Medicine, McGill University, Montreal, Quebec, Canada

${ }^{5}$ Research Center, Institut de Cardiologie de Montréal, Montreal, Quebec, Canada ${ }^{6}$ Institute for Health System Solutions and Virtual Care, Women's College Hospital Institute for Health System Solutions and Virtual Care, Women's College Hospital, Toronto, Ontario, Canada

${ }^{7}$ Psychiatry Department, Faculty of Medicine, Université de Montréal, Montreal, Quebec, Canada

\section{Twitter Guillaume Fontaine @_GFontaine and Geneviève Rouleau @GeRouleau}

Acknowledgements This study protocol was developed as part of the $\mathrm{PhD}$ training of GC and for which she received scholarships from the following: Université de Montréal Hospital Center Foundation, Quebec's Ministry of Education and Higher Education Scholarship Program, Fonds de recherche du Québec - Société et Culture (FRQSC), Université de Montréal Research Chair in Innovative Nursing Practices, Université de Montréal Center for Innovation in Nursing Education and Équipe FUTUR (FRQSC).

Contributors GC conceptualised the study, designed the study, developed the study protocol and wrote the first draft of this manuscript. JC, JP and DJ-A helped to conceptualise the study, design the study and made comments on the first draft of this manuscript. JC is principal supervisor, and JP and DJ-A are cosupervisors for the PhD training of GC. PP and LB are discipline-specific members of GC thesis committee and have contributed to conceptualise and design specific aspects of the study. PP, LB, GF and GR helped develop the study protocol and made comments on the draft. All authors critically reviewed, edited and approved the final manuscript. The recruitment of participants for the study, data collection, management and analysis and the final interpretation of the study results will be led by GC. SD is medical director of the ECHO program on concurrent disorder management at the Université de Montréal Hospital Center, and he assisted GC in developing the measuring instruments for the study. GC will have access to the quantitative online database and the qualitative raw data. JC, JP and DJ-A will assist GC in analysing the data, interpretating the study results and disseminating the study results.

Funding The authors have not declared a specific grant for this research from any funding agency in the public, commercial or not-for-profit sectors.

Competing interests None declared.

Patient consent for publication Not required.

Provenance and peer review Not commissioned; externally peer reviewed.

Supplemental material This content has been supplied by the author(s). It has not been vetted by BMJ Publishing Group Limited (BMJ) and may not have been peer-reviewed. Any opinions or recommendations discussed are solely those of the author(s) and are not endorsed by BMJ. BMJ disclaims all liability and responsibility arising from any reliance placed on the content. Where the content includes any translated material, BMJ does not warrant the accuracy and reliability of the translations (including but not limited to local regulations, clinical guidelines, terminology, drug names and drug dosages), and is not responsible for any error and/or omissions arising from translation and adaptation or otherwise.

Open access This is an open access article distributed in accordance with the Creative Commons Attribution Non Commercial (CC BY-NC 4.0) license, which permits others to distribute, remix, adapt, build upon this work non-commercially, and license their derivative works on different terms, provided the original work is properly cited, appropriate credit is given, any changes made indicated, and the use is non-commercial. See: http://creativecommons.org/licenses/by-nc/4.0/.

ORCID iDs

Gabrielle Chicoine http://orcid.org/0000-0003-3179-5806

Guillaume Fontaine http://orcid.org/0000-0002-7806-814X

Geneviève Rouleau http://orcid.org/0000-0003-1093-6577

\section{REFERENCES}

1 American Psychiatric Association [APA]. Diagnostic and statistical manual of mental disorders: DSM-5. 5 edn. Washington, DC: American Psychiatric Publishing, 2013.

2 Kessler RC, Chiu WT, Demler O, et al. Prevalence, severity, and comorbidity of 12-month DSM-IV disorders in the National comorbidity survey replication. Arch Gen Psychiatry 2005;62:617-27.

3 Urbanoski K, Inglis D, Veldhuizen S. Service use and unmet needs for substance use and mental disorders in Canada. Can J Psychiatry 2017;62:551-9.

4 World Health Organization [WHO]. Mental health action plan, 2013. Available: https://www.who.int/publications/i/item/9789241506021 [Accessed 21 Dec 2020].

5 Fleury M-J, Grenier G, Bamvita J-M, et al. Profiles associated respectively with substance dependence only, mental disorders only and co-occurring disorders. Psychiatr Q 2015;86:355-71.

6 Khan S. Troubles concomitants de santé mentale et de consommation d'alcool ou de drogues au Canada (Rapports sur la santé): Statistique Canada, 2017. Available: http://www.statcan.gc. ca/pub/82-003-x/2017008/article/54853-fra.pdf [Accessed 21 Dec 2020].

7 Crockford D, Addington D. Canadian schizophrenia guidelines: schizophrenia and other psychotic disorders with coexisting substance use disorders. Can J Psychiatry 2017;62:624-34.

8 Ghabrash MF, Bahremand A, Veilleux M, et al. Depression and outcomes of methadone and buprenorphine treatment among people with opioid use disorders: a literature review. J Dual Diagn 2020;16:1-17.

9 O'Neill S, Lapalme M, Camiré M. Modèles de dispensation des soins et services aux personnes présentant des troubles concomitants de santé mentale et de dépendance: Institut Nationale d'Excellence en Santé et en Services Sociaux [INESSS], 2016. Available: https:// www.inesss.qc.ca/fileadmin/doc/INESSS/Rapports/ServicesSociaux/ INESSS_Avis_Dispensation_soins_services_troubles_concomitants. pdf [Accessed 15 Dec 2020].

10 National Institute for Health and Care Excellence [NICE]. Coexisting severe mental illness and substance misuse: community health and social care services, 2016. Available: https://www.nice.org.uk/ guidance/ng58 [Accessed 21 Dec 2020].

11 Padwa H, Guerrero EG, Braslow JT, et al. Barriers to serving clients with co-occurring disorders in a transformed mental health system. Psychiatr Serv 2015;66:547-50.

12 Substance Abuse and Mental Health Services Administration [SAMHSA]. Behavioral health trends in the United States: results from the 2014 national survey on drug use and health, 2014. Available: https://www.samhsa.gov/data/sites/default/files/NSDUHFRR1-2014/NSDUH-FRR1-2014.pdf [Accessed 7 Jan 2020].

13 Levesque J-F, Sutherland K. Combining patient, clinical and system perspectives in assessing performance in healthcare: an integrated measurement framework. BMC Health Serv Res 2020;20:23.

14 Canadian Institute for Health Information [CIHI]. Health care cost drivers: the facts, 2011. Available: https://secure.cihi.ca/free products/health_care_cost_drivers_the_facts_en.pdf [Accessed 16 Apr 2020].

15 Garrod E, Jenkins E, Currie LM, et al. Leveraging nurses to improve care for patients with concurrent disorders in inpatient mental health settings: a scoping review. J Dual Diagn 2020;16:1-16.

16 Wheeler A, Crozier M, Robinson G. Assessing and responding to hazardous and risky alcohol and other drug use: the practice, knowledge and attitudes of staff working in mental health services. Drugs: Education, Prevention and Policy 2014;21:234-43.

17 Roncero C, Szerman N, Terán A, et al. Professionals' perception on the management of patients with dual disorders. Patient Prefer Adherence 2016;10:1855-68. 
18 Coloma-Carmona A, Carballo JL, Tirado-González S. Barriers for identification and treatment of problem drinkers in primary care. Gac Sanit 2017;31:95-99.

19 Wener P, Woodgate RL. Looking for Help: Primary Care Providers' Need for Collaboration to Deliver Primary Mental Healthcare Services. Can J Commun Ment Health 2017;36:29-39.

20 Somers JM, Moniruzzaman A, Rezansoff SN, et al. The prevalence and geographic distribution of complex co-occurring disorders: a population study. Epidemiol Psychiatr Sci 2016;25:267-77.

21 Coombes L, Wratten A. The lived experience of community mental health nurses working with people who have dual diagnosis: a phenomenological study. J Psychiatr Ment Health Nurs 2007;14:382-92.

22 Pinderup P, Thylstrup B, Hesse M. Critical review of dual diagnosis training for mental health professionals. Int $J$ Ment Health Addict 2016;14:856-72.

23 Behilak S, AS-e A. The relationship between burnout and job satisfaction among psychiatric nurses. J Nurs Educ Pract 2020;10.

24 Dawood E, Mitsu R, Monica A. Perceived psychiatric nurses job stress: a cross sectional study. IOSR Journal of Nursing and Health Science 2017;06:37-47.

25 Havens DS, Gittell JH, Vasey J. Impact of relational coordination on nurse job satisfaction, work engagement and burnout: achieving the quadruple aim. J Nurs Adm 2018;48:1.

26 Priester MA, Browne T, lachini A, et al. Treatment access barriers and disparities among individuals with co-occurring mental health and substance use disorders: an integrative literature review. J Subst Abuse Treat 2016:61:47-59.

27 Molina-Mula J, González-Trujillo A, Simonet-Bennassar M. Emergency and Mental Health Nurses' Perceptions and Attitudes towards Alcoholics. Int J Environ Res Public Health 2018;15:1733.

28 van Boekel LC, Brouwers EPM, van Weeghel J, et al. Healthcare professionals' regard towards working with patients with substance use disorders: comparison of primary care, general psychiatry and specialist addiction services. Drug Alcohol Depend 2014;134:92-8.

29 Petrakis M, Robinson R, Myers K, et al. Dual diagnosis competencies: a systematic review of staff training literature. Addict Behav Rep 2018;7:53-7.

30 Pinderup P. Improving the knowledge, attitudes, and practices of mental health professionals regarding dual diagnosis treatment - a mixed methods study of an intervention. Issues Ment Health Nurs 2018;39:292-303.

31 Savic M, Best D, Manning V, et al. Strategies to facilitate integrated care for people with alcohol and other drug problems: a systematic review. Subst Abuse Treat Prev Policy 2017;12:19.

32 Pepin J, Goudreau J, Lavoie P, et al. A nursing education research framework for transformative learning and interdependence of academia and practice. Nurse Educ Today 2017;52:50-2.

33 Arora S, Kalishman S, Dion D, et al. Partnering urban academic medical centers and rural primary care clinicians to provide complex chronic disease care. Health Aff 2011;30:1176-84

34 Coventry TH, Maslin-Prothero SE, Smith G. Organizational impact of nurse supply and workload on nurses continuing professional development opportunities: an integrative review. J Adv Nurs 2015;71:2715-27.

35 McBain RK, Sousa JL, Rose AJ, et al. Impact of Project echo models of medical Tele-Education: a systematic review. J Gen Intern Med 2019;34:2842-57.

36 Zhou C, Crawford A, Serhal E, et al. The impact of Project echo on participant and patient outcomes: a systematic review. Acad Med 2016;91:1439-61.

37 Beste LA, Glorioso TJ, Ho PM, et al. Telemedicine specialty support promotes hepatitis $C$ treatment by primary care providers in the Department of Veterans Affairs. Am J Med 2017;130:432-8.

38 Ní Cheallaigh C, O'Leary A, Keating S, et al. Telementoring with project echo: a pilot study in Europe. BMJ Innov 2017;3:144-51.

39 Furlan $A D$, Zhao J, Voth J, et al. Evaluation of an innovative teleeducation intervention in chronic pain management for primary care clinicians practicing in underserved areas. $J$ Telemed Telecare 2019;25:484-92.

40 De Witt Jansen B, Brazil K, Passmore P, et al. Evaluation of the impact of telementoring using $\mathrm{ECHO} \odot$ technology on healthcare professionals' knowledge and self-efficacy in assessing and managing pain for people with advanced dementia nearing the end of life. BMC Health Serv Res 2018;18:228.

41 Carlin L, Zhao J, Dubin R, et al. Project echo Telementoring intervention for managing chronic pain in primary care: insights from a qualitative study. Pain Med 2018;19:1140-6.

42 Fisher E, Hasselberg M, Conwell Y, et al. Telementoring primary care clinicians to improve geriatric mental health care. Popul Health Manag 2017;20:342-7.
43 Moore AB, Krupp JE, Dufour AB, et al. Improving transitions to Postacute care for elderly patients using a novel Video-Conferencing program: ECHO-Care transitions. Am J Med 2017;130:1199-204.

44 Komaromy M, Bartlett J, Manis K, et al. Enhanced primary care treatment of behavioral disorders with echo case-based learning. Psychiatr Serv 2017;68:873-5.

45 Sockalingam S, Arena A, Serhal E, et al. Building provincial mental health capacity in primary care: an evaluation of a project echo mental health program. Acad Psychiatry 2018;42:451-457.

46 Frank JW, Carey EP, Fagan KM, et al. Evaluation of a telementoring intervention for pain management in the Veterans health administration. Pain Med 2015;16:1090-100.

47 Mehrotra K, Chand P, Bandawar M, et al. Effectiveness of NIMHANS echo blended tele-mentoring model on integrated mental health and addiction for counsellors in rural and underserved districts of Chhattisgarh, India. Asian J Psychiatr 2018;36:123-7.

48 Faherty LJ, Rose AJ, Chappel A, et al. Assessing and expanding the evidence base for project echo and ECHO-Like models: findings of a technical expert panel. J Gen Intern Med 2020;35:899-902.

49 Albarqouni L, Glasziou P, Hoffmann T. Completeness of the reporting of evidence-based practice educational interventions: a review. Med Educ 2018;52:161-70.

50 White C, Mcllfatrick S, Dunwoody L, et al. Supporting and improving community health services-a prospective evaluation of echo technology in community palliative care nursing teams. BMJ Support Palliat Care 2019;9:202-8.

51 Dowling MJ, Payne C, Larkin P, et al. Does an interactive, Teleconference-Delivered, palliative care lecture series improve nursing home staff confidence? J Palliat Med 2020;23:179-83.

52 Hacking I. The social construction of what? Cambridge, MA: Harvard University Press, 1999

53 Pluye P, Gagnon M-P, Griffiths F, et al. A scoring system for appraising mixed methods research, and concomitantly appraising qualitative, quantitative and mixed methods primary studies in mixed studies reviews. Int J Nurs Stud 2009;46:529-46.

54 Pluye P, Kaur N, Granikov V. Mixing phases, results and data in patient oriented research. Int J Mult Res Approaches 2018;10:41-56.

55 Gregor S. The nature of theory in information systems. MIS Quarterly 2006;30:611-42.

56 Moore DE, Green JS, Gallis HA. Achieving desired results and improved outcomes: integrating planning and assessment throughout learning activities. J Contin Educ Health Prof 2009:29:1-15

57 Tardif J. L'évaluation des compétences: Documenter Le parcours de développement: Montreal. Canada: Chenelière-éducation, 2006.

58 Sandberg J, Alvesson M. Ways of constructing research questions: gap-spotting or problematization? Organization 2011;18:23-44.

59 O'Cathain A, Murphy E, Nicholl J. The quality of mixed methods studies in health services research. $J$ Health Serv Res Policy 2008;13:92-8.

60 von Elm E, Altman DG, Egger M, et al. Strengthening the reporting of observational studies in epidemiology (STROBE) statement: guidelines for reporting observational studies. BMJ 2007;335:806-8.

61 Tong A, Sainsbury P, Craig J. Consolidated criteria for reporting qualitative research (COREQ): a 32-item checklist for interviews and focus groups. Int J Qual Health Care 2007;19:349-57.

62 Phillips AC, Lewis LK, McEvoy MP, et al. Development and validation of the Guideline for reporting evidence-based practice educational interventions and teaching (GREET). BMC Med Educ 2016;16:237.

63 Creswell JW, Plano Clark VL. Designing and conducting mixed methods research. 3 edn. Los Angeles, CA: SAGE Publications, 2018.

64 O'Cathain A, Murphy E, Nicholl J. Three techniques for integrating data in mixed methods studies. BMJ 2010;341:c4587.

65 Thorne S. Interpretive description. New York, NY: Routledge, 2008.

66 Socolovsky C, Masi C, Hamlish T, et al. Evaluating the role of key learning theories in echo: a telehealth educational program for primary care providers. Prog Community Health Partnersh 2013;7:361-8.

67 Arora S, Thornton K, Murata G, et al. Outcomes of treatment for hepatitis $C$ virus infection by primary care providers. N Engl J Med 2011;364:2199-207.

68 McLoughlin C, Patel KD, O'Callaghan T, et al. The use of virtual communities of practice to improve interprofessional collaboration and education: findings from an integrated review. $J$ Interprof Care 2018:32:136-42.

69 Grove SK, Burns N, Gray J. The practice of nursing research: appraisal, synthesis, and generation of evidence. 8 edn. St-Louis, MO: Elsevier, 2017 
70 Thompson Burdine J, Thorne S, Sandhu G. Interpretive description: a flexible qualitative methodology for medical education research. Med Educ 2021;55:336-43.

71 Paillé P, Mucchielli A. L'analyse thématique. In: Paillé P, Mucchielli A, eds. L'analyse qualitative en sciences humaines et sociales. 4 edn. Malakoff, France: Armand Colin, 2016: 235-312.

72 Birt L, Scott S, Cavers D, et al. Member checking: a tool to enhance Trustworthiness or merely a NOD to validation? Qual Health Res 2016;26:1802-11.

73 Johnson RE, Grove AL, Clarke A. Pillar integration process: a joint display technique to integrate data in mixed methods research. J Mix Methods Res 2017;13.

74 Guetterman TC, Fetters MD, Creswell JW. Integrating quantitative and qualitative results in health science mixed methods research through joint displays. Ann Fam Med 2015;13:554-61.

75 Morgan DL. Practical strategies for combining qualitative and quantitative methods: applications to health research. Qual Health Res 1998;8:362-76.

76 Wendler MC. Triangulation using a meta-matrix. J Adv Nurs 2001;35:521-5

77 Moffatt S, White M, Mackintosh J, et al. Using quantitative and qualitative data in health services research - what happens when mixed method findings conflict? [ISRCTN61522618]. BMC Health Serv Res 2006;6:28.

78 Frenk J, Chen L, Bhutta ZA, et al. Health professionals for a new century: transforming education to strengthen health systems in an interdependent world. Lancet 2010;376:1923-58.

79 Canadian Institutes of Health Research [CIHR], Natural Sciences and Engineering Research Council of Canada [NSERCC], Social Sciences and Humanities Research Council [SSHRC]. Tri-Council policy statement: ethical conduct for research involving humans (TCPS2), 2018. Available: https://ethics.gc.ca/eng/documents/tcps2-2018-eninteractive-final.pdf [Accessed 26 Dec 2020]
80 Chicoine G. How are we conducting and reporting mixed methods research in nursing sciences?: a commentary on Seah et al. (2018) publication. Arch Psychiatr Nurs 2018;32:784-5.

81 Arora S, Kalishman S, Thornton K, et al. Expanding access to hepatitis $\mathrm{C}$ virus treatment--Extension for Community Healthcare Outcomes $(\mathrm{ECHO})$ project: disruptive innovation in specialty care. Hepatology 2010;52:1124-33.

82 Watson HE, Maclaren W, Shaw F. Effective interventions unit: measuring staff attitudes to people with drug problems: the development of a tool (drug misuse research programme). Glasgow, Scottish: Glasgow Caledonian University, 2003. https://www. webarchive.org.uk/wayback/archive/20180515184547/http://www. gov.scot/Publications/2003/08/17735/23437

83 Munro A, Watson HE, McFadyen A. Assessing the impact of training on mental health nurses' therapeutic attitudes and knowledge about co-morbidity: a randomised controlled trial. Int J Nurs Stud 2007;44:1430-8.

84 Crowe TP, Kelly P, Pepper J, et al. Service based internship training to prepare workers to support the recovery of people with cooccurring substance abuse and mental health disorders. Int $J$ Ment Health Addict 2013;11:269-80.

85 Pinderup P. Training changes professionals' attitudes towards dual diagnosis. Int J Ment Health Addict 2017;15:53-62.

86 Bandura A. Guide for constructing self-efficacy scales. In: Pajares F, Urdan T, eds. Self-Efficacy beliefs in adolescents. Greenwich, Connectivut: Information Age Publishing, 2006: 307-37.

87 Hughes E. Closing the gap: a capability framework for working effectively with people with combined mental health and substance use problems (dual diagnosis. Mansfield, UK: University of Lincoln, Centre for Clinical and Academic Workforce Innovation, 2006. http:// eprints.lincoln.ac.uk/id/eprint/729/1/uoa12eh05.pdf 
Correction: Impact of a videoconferencing educational programme for the management of concurrent disorders on nurses' competency development and clinical practice: protocol for a convergent mixed methods study

Chicoine G, Côté J, Pepin J, et al Impact of a videoconferencing educational programme for the management of concurrent disorders on nurses' competency development and clinical practice: protocol for a convergent mixed methods studyBMJ Open 2021;11:e042875. doi: ${ }^{10.1136} /$ bmjopen-2020-042875

This article was previously published with an error.The author name Pepin was published as Pépin.

Open access This is an open access article distributed in accordance with the Creative Commons Attribution Non Commercial (CC BY-NC 4.0) license, which permits others to distribute, remix, adapt, build upon this work non-commercially, and license their derivative works on different terms, provided the original work is properly cited, appropriate credit is given, any changes made indicated, and the use is non-commercial. See: http://creativecommons.org/licenses/by-nc/4.0/.

C Author(s) (or their employer(s)) 2021. Re-use permitted under CC BY-NC. No commercial re-use. See rights and permissions. Published by BMJ.

BMJ Open 2021;11:e042875corr1. doi:10.1136/bmjopen-2020-042875corr1

A) Check for updates 